\title{
Lectura y Cálculo de Facturación en Sitio a Través de Dispositivos Móviles
}

\section{Reading and Calculating Billing Through Mobile Devices}

\section{Moreno Pilar Alexandra ${ }^{1}$, Flechas Becerra Yaneth², Rojas López Carlos Alberto ${ }^{3}$}

\author{
${ }^{1}$ Escuela de Ciencias Básicas, Tecnología e Ingeniería, Universidad Nacional Abierta \\ y a Distancia, Acacias, Colombia, pilar.moreno@unad.edu.co \\ ${ }^{2}$ Escuela de Ciencias Básicas, Tecnología e Ingeniería, Universidad Nacional Abierta \\ y a Distancia, Acacias, Colombia, hilda.flechas@unad.edu.co \\ ${ }^{3}$ Escuela de Ciencias Básicas, Tecnología e Ingeniería, Universidad Nacional Abierta \\ y a Distancia, Acacias, Colombia, carlosa.rojas@unad.edu.co
}

Recibido: 22/08/2012 • Aprobado.15/11/2012

\section{RESUMEN}

Este artículo describe ampliamente el análisis, diseño y desarrollo del sistema tipo utilitario, denominado "Lectura y cálculo de facturación en sitio a través de dispositivos móviles". La aplicación está orientada a empresas prestadoras de servicios públicos; en primera instancia, servicios de acueducto, para realizar parte del proceso de facturación "en sitio" a través de celulares o cualquier tipo de dispositivos móviles, compatibles con Android. Permite realizar la toma de lecturas del consumo del servicio, registrar novedades de aforo, actualizar y controlar en línea la información correspondiente de usuarios y facturación. Esta tecnología se considera, como tal, un método de facturación en sitio, ya que a través de internet se conecta con la base de datos de la empresa, enviando y recibiendo información actualizada, con la que se hace el cálculo de la facturación correspondiente al periodo de la lectura, beneficiando no solo al cliente, sino a la misma empresa generadora del servicio.

Palabras clave: cálculo de facturación, facturación en línea, lectura en sitio, programación con Android, facturación en sitio

\section{Abstract}

This article broadly describes the analysis, design and development of the system utilitarian, called "Reading and billing calculation site through mobile devices." The application is oriented Public Services Companies, first water services, to perform part of the billing process "in place" through phones or any mobile devices compatible with Android. Will enable you to take readings of service consumption, recording new gauging, online update and control the information for users and turnover. This technology is considered as such one site billing method as through Internet is connected with the database of the company, sending and receiving date information, which makes the calculation of the billing for the reading period, bringing benefits to the client and the service generating company.

Keywords: calculation billing, online billing, site reading, programming Android, in site billing 


\section{INTRODUCCIÓN}

La aplicación que se presenta es una solución móvil que permite que a un usuario se le procese su factura al instante, luego de la toma de lectura en su contador de agua.

Es realmente una nueva solución tecnológica de software para empresas generadoras y prestadoras de servicios públicos, la cual corre en dispositivos móviles compatibles con Android, como los que manejan o deben manejar sus funcionarios o contratistas encargados de tomar las lecturas. Esta tecnología es indispensable y está lista para usarse como método de facturación en sitio.

El aplicativo le permite a un contratista, operario o aforador de las empresas de servicios de agua, hacer la lectura de cada uno de los medidores, siguiendo las rutas previamente establecidas y procesar en ese mismo instante la factura por el consumo hasta ese momento; para un futuro, se piensa complementar la aplicación con la impresión de la misma para entregársela al usuario.

La aplicación está orientada a empresas prestadoras de servicios públicos, con el fin de realizar parte del proceso de facturación "en sitio" a través de celulares o cualquier tipo de dispositivo móvil, compatible con Android. Desde cada vivienda, es decir, en el sitio que se origina la información, es posible tomar las lecturas del consumo del servicio, registrar novedades de aforo, y actualizar y controlar en línea la información correspondiente de usuarios y facturación.

Además, está planteada como un utilitario o módulo independiente que, si es necesario, se puede enlazar con sistemas software de servicios públicos en determinado momento y con ciertos ajustes, según el sistema de facturación.

\section{Metodología}

\section{A. Antecedentes}

Realizando una revisión detallada de las aplicaciones disponibles en línea, que puedan ejecutar las mismas tareas, acciones y/o usos, que se propone desarrollar en este Proyecto, no se encontró descrita ninguna aplicación igual. Tomando como parámetros de búsqueda palabras clave como "cálculo de facturación" o "facturación en línea" o "lectura en sitio" o "facturación en sitio" se encontraron funcionalidades que apuntan a realizar facturación, pero ninguna de servicios públicos y mucho menos de toma de lecturas en línea; en su mayoría, son utilidades que realizan facturación en línea para compras de productos de empresas comerciales, por lo que no se puede establecer como tal un paralelo con lo que se propone en este trabajo. Dichas búsquedas se realizaron en Google Play.

En la tabla 1 se presenta un cuadro comparativo con la revisión de aplicaciones investigadas: 
TABLA I

Cuadro Comparativo de Aplicaciones Analizadas.

\begin{tabular}{|c|c|c|}
\hline \multirow{2}{*}{$\begin{array}{l}\text { Caracterís- } \\
\text { ticas } \\
\text { /Parámetros }\end{array}$} & Aplicación 1 & Aplicación 2 \\
\hline & $\begin{array}{l}\text { EGENCIA } \\
\text { MOBILE }\end{array}$ & $\begin{array}{c}\text { Movalnvoice } \\
\text { Facturación-App }\end{array}$ \\
\hline Descripción & $\begin{array}{l}\text { Se centra en la } \\
\text { tecnología de } \\
\text { viajes, la aten- } \\
\text { ción a los viaje- } \\
\text { ros y en prestar } \\
\text { sus servicios en } \\
\text { todo el mundo. }\end{array}$ & $\begin{array}{l}\text { Orientada al sec- } \\
\text { tor del comercio } \\
\text { en general, para } \\
\text { pequeñas empre- } \\
\text { sas o trabajado- } \\
\text { res autónomos. }\end{array}$ \\
\hline Objetivo & $\begin{array}{l}\text { Realizar alertas } \\
\text { sobre vuelos y } \\
\text { destinos, factu- } \\
\text { ración en línea y } \\
\text { soporte a clien- } \\
\text { tes, aplicación } \\
\text { eficaz, inteligen- } \\
\text { te y segura. }\end{array}$ & $\begin{array}{l}\text { Realizar factu- } \\
\text { ras, pedidos y } \\
\text { presupuestos. } \\
\text { Además permite } \\
\text { crear articulos y } \\
\text { clientes }\end{array}$ \\
\hline $\begin{array}{l}\text { Sector del } \\
\text { mercado }\end{array}$ & Aerolíneas & Comercial \\
\hline $\begin{array}{l}\text { Facturación } \\
\text { en línea }\end{array}$ & SI & SI \\
\hline $\begin{array}{l}\text { Lectura de } \\
\text { consumos } \\
\text { en línea }\end{array}$ & NO & NO \\
\hline $\begin{array}{l}\text { Tipo de } \\
\text { factura }\end{array}$ & Factura de venta & Factura de venta \\
\hline $\begin{array}{l}\text { Genera } \\
\text { factura }\end{array}$ & $\mathrm{SI}$ & SI \\
\hline $\begin{array}{l}\text { Facturación } \\
\text { de servicios } \\
\text { públicos }\end{array}$ & NO & NO \\
\hline $\begin{array}{l}\text { Toma lectu- } \\
\text { ras en línea }\end{array}$ & NO & NO \\
\hline $\begin{array}{l}\text { Permite } \\
\text { integración } \\
\text { con otros } \\
\text { sistemas }\end{array}$ & NO & NO \\
\hline Escalable & NO & NO \\
\hline $\begin{array}{l}\text { Permite per- } \\
\text { sonalización }\end{array}$ & NO & NO \\
\hline $\begin{array}{l}\text { Permite } \\
\text { ajustes }\end{array}$ & NO & NO \\
\hline
\end{tabular}

Los parámetros tomados para la comparación corresponden a las características que debía contener y que efectivamente presenta el aplicativo desarrollado.
Ya, a manera comercial, existen algunos sistemas de facturación en sitio que ofrecen diferentes compañías de software para sus clientes; estas son empresas de servicios públicos que poseen sus sistemas de facturación y desean implementar la toma de lecturas en tiempo real, disminuyendo costos y aumentando la calidad del servicio a sus usuarios.

Algunas empresas de desarrollo de software que ofrecen este tipo de servicio son: Sysman Software [1], e infotrack [2].

\section{B. Análisis de requerimientos para el aplicativo}

El utilitario desarrollado, diseñado para Android, cumple con una serie de características específicas, que describen los alcances de su aplicación:

1. Manejo de seguridad para acceder al módulo, tanto para los usuarios como para el administrador, registrando contraseñas para cada uno.

2. Facilidad en la toma de lecturas del servicio de acueducto en el sitio.

3. Efectividad en la toma de lecturas en fechas reales, ya que se toma la lectura en sitio.

4. Control de las novedades de aforo que se consideran en la toma de lectura del medidor.

5. Facilidad para la actualización de la información de toma de lecturas, que será utilizada para una empresa de Servicios Públicos.

Igualmente, se señalan algunas limitaciones, que corresponden a funcionalidades que la aplicación no contempla realizar:

- Expedir facturas físicas (impresión de facturas).

- Enviar facturas al correo electrónico de los usuarios.

- Realizar la precrítica de lecturas en el momento de la toma de lectura. 


\section{Diseño del sistema}

En este apartado se presenta, en primer lugar, el esquema general del funcionamiento de la aplicación, el cual identifica el contexto, los usuarios, los sistemas y las interacciones entre ellos, dando así una visión general y consolidada de lo que realiza el sistema desarrollado Fig. 1.

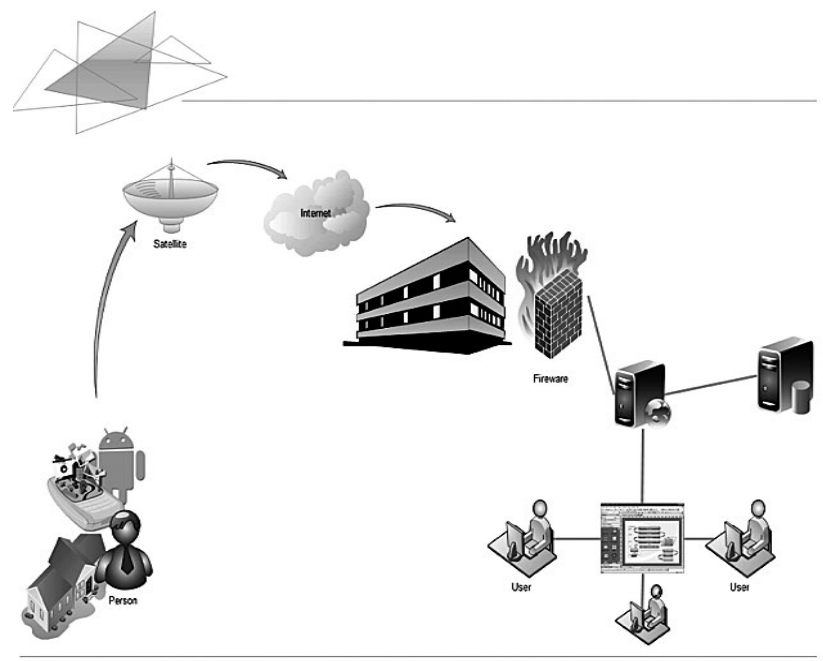

Fig. 1 Esquema general del sistema

En segundo lugar, se describe paso a paso el proceso seguido en el diseño y desarrollo del utilitario "Lectura y cálculo de facturación en sitio a través de dispositivos móviles", bajo sistema operativo Android con las bibliotecas disponibles de Java, bajo el framework Java.

Este proceso empieza una vez se aborda la etapa de análisis de requerimientos, en la cual se establecen de manera clara los alcances y limitaciones que tiene la aplicación; sobre esta se realiza el diseño y modelamiento del sistema. Para ello se diagramaron, a través de UML, los esquemas básicos del sistema, así:

Un único diagrama de caso de uso, en donde se muestra la funcionalidad e interacciones para la aplicación, y que documenta, desde un enfoque sencillo y práctico, el comportamiento del sistema; allí se determinan los requisitos funcionales del mismo, es decir, se representan las tareas que este puede ejecutar. En la Fig. 2 están claramente definidos los elementos que integran el sistema en línea, tales como entradas, salidas, procesos e interacciones.

Su ventaja principal radica en la facilidad para interpretarlo, lo que lo hace especialmente útil en la comunicación con el cliente, para así verificar que lo que él necesita es lo que realmente se diseñó.

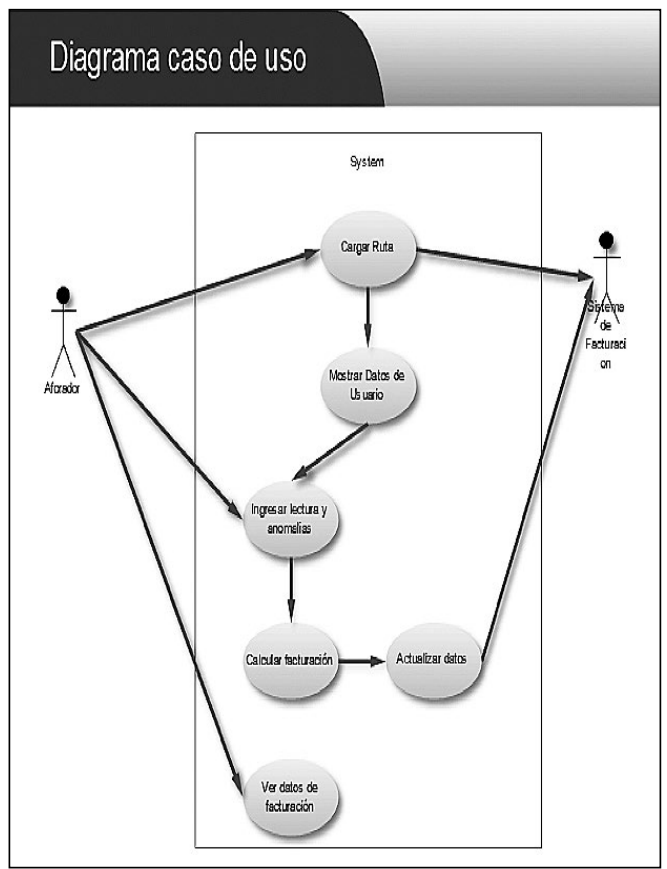

Fig. 2 Diagrama de caso de uso

Un diagrama de clases, que describe la estructura del sistema mostrando sus clases, atributos y las relaciones entre ellos Fig. 3 .
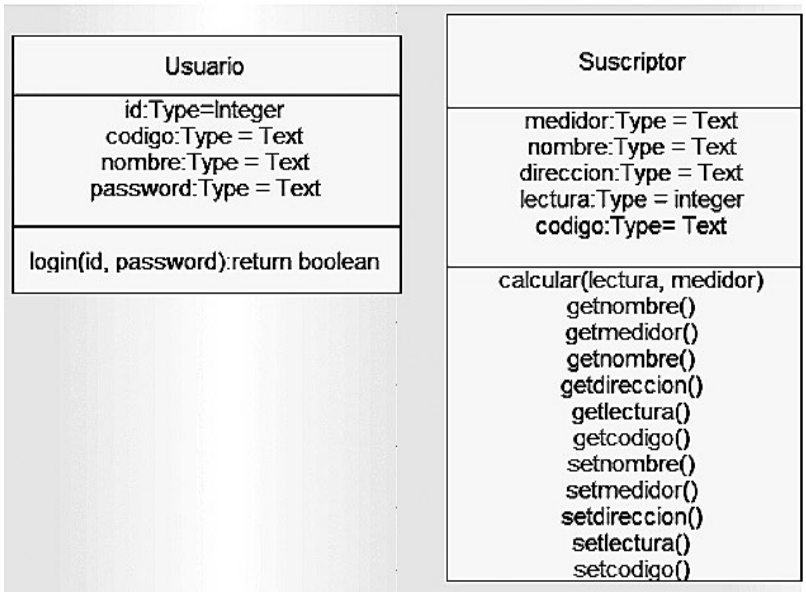

Fig. 3 Diagrama de clases 
Un diagrama de secuencia, usado para modelar la interacción entre los objetos del sistema según UML Fig.4.

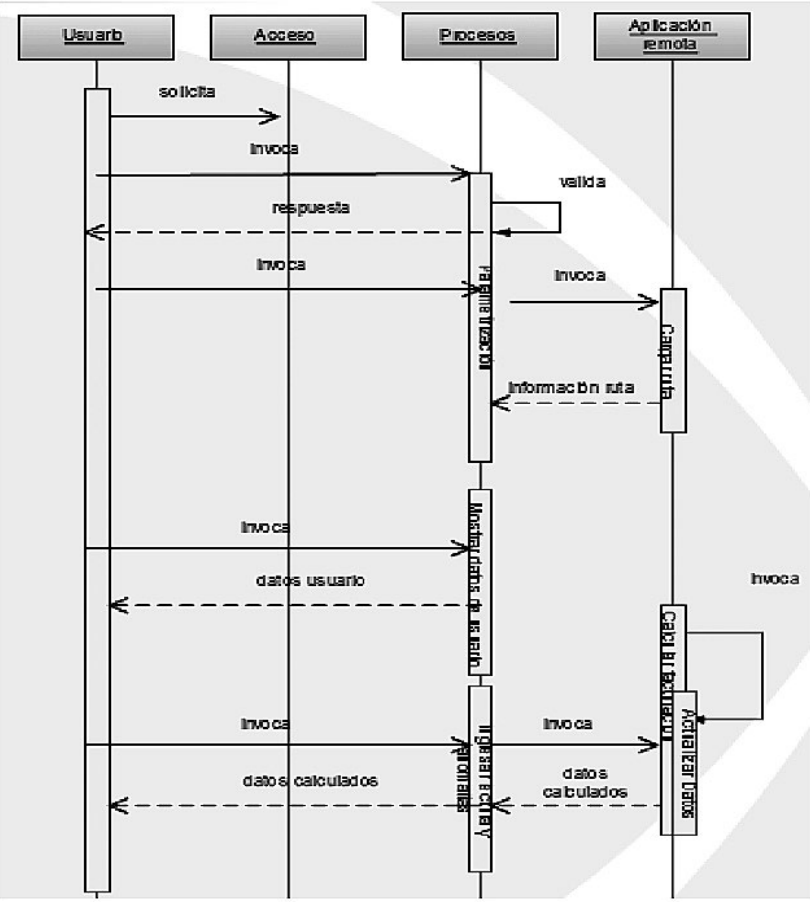

Fig. 4 Diagrama de secuencia

Un diagrama de comunicación, en el cual se establecen las interacciones entre los objetos o partes en términos de mensajes en secuencia Fig. 5

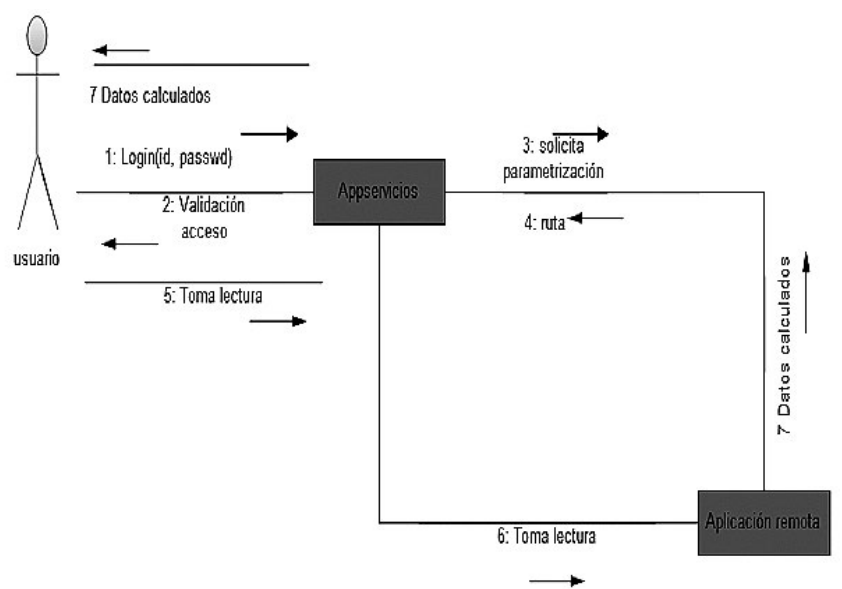

Fig. 5 Diagrama de comunicación

Posterior al modelamiento del sistema con UML, se continuó con la creación del proyecto en el IDE Eclipse. Para ello, fue necesario la revisión de documentación acerca de la instalación de herramientas básicas para desarrollar en Android; entre ellas: IDE Eclipse, JDK, SDK, NDK y plugin ADT. Una vez instaladas y configuradas las herramientas necesarias se empezó a revisar y a estudiar pequeños programas de ejemplo, empezando por "Hola mundo", hasta llegar a algunos un poco más complejos con manejo de menús, listas de opciones y captura de datos.

Dicha contextualización fue necesaria debido a que es el primer desarrollo bajo Android que realizan los programadores del sistema; así se inicia, entonces, con el diseño de las primeras pantallas y funcionalidades de la aplicación, contenedores, servicios y layouts.

En la Fig. 6 se observa como ejemplo en un dispositivo Android la forma como se visualiza en el menú el acceso a la aplicación a través de un ícono con una imagen alusiva al aplicativo, la cual es una llave llenando un vaso de agua (señalada con el círculo rojo). También se presenta el menú del proyecto Android en el IDE Eclipse, sus layout, dependencias y recursos.

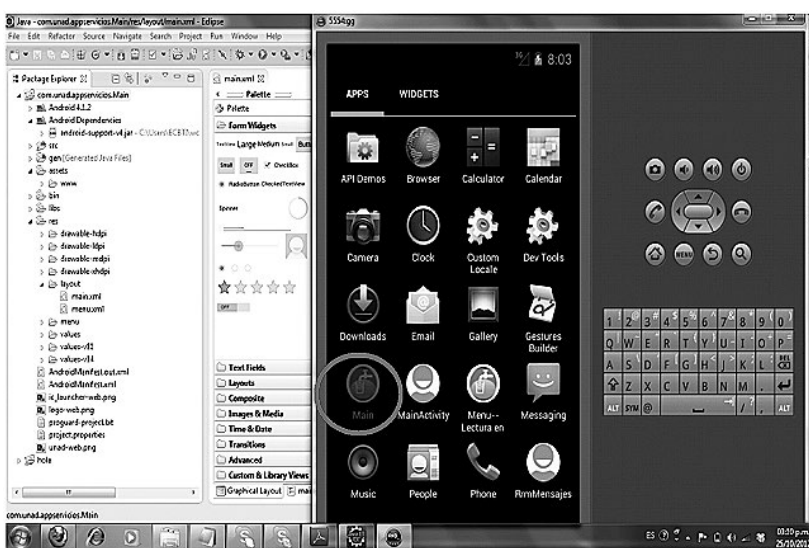

Fig. 6 Pantalla Main del proyecto

Para iniciar la aplicación "Lectura en sitio", en la pantalla inicial del dispositivo se busca y aplica el ícono con la figura descrita y el nombre "Main"; la ventana de la Fig. 7, evidencia la primera pantalla de la aplicación, correspondiente al logueo e ingreso del usuario. 


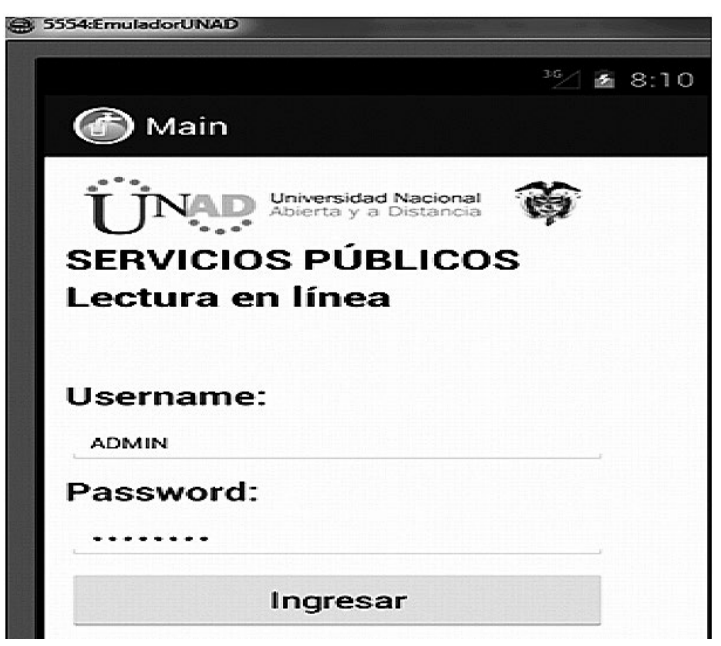

Fig. 7 Logueo e ingreso de usuarios

Cada password puede estar conformado por letras y números. No debe superar los 40 caracteres y se debe escribir teniendo en cuenta la configuración del teclado del dispositivo móvil.

Cuando la pantalla descrita anteriormente aparece en el dispositivo, el cursor está posicionado en el cuadro de texto Username, de manera que se puede digitar o teclear el usuario inmediatamente; luego, con la tecla enter o con las teclas de navegación se ubica el cursor en el cuadro de texto Password para, de igual forma, digitar la clave de acceso. Al finalizar, se debe presionar el botón Ingresar. En el caso de que la clave sea incorrecta aparecerá un mensaje advirtiendo de este error.

Una vez validada satisfactoriamente la clave, aparece la ventana con el menú principal del sistema Fig 8.

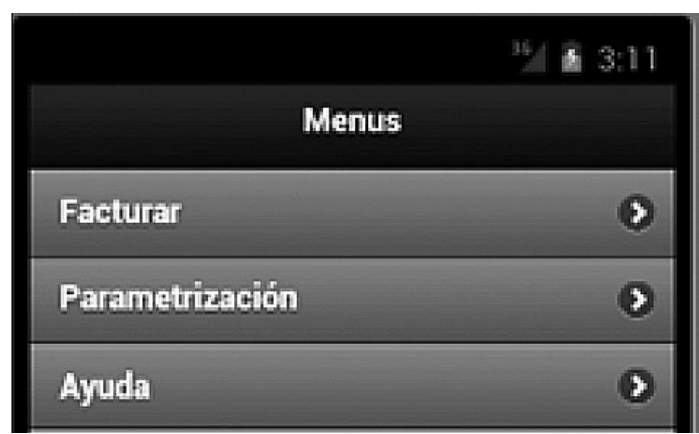

Fig. 8 Menú principal
Para mejorar la apariencia, optimizar la navegabilidad y lograr mayor espacio de trabajo se ocultó la barra superior en la que aparece el logo y el nombre de la aplicación.

La opción principal se denomina "Facturar". Para poder trabajar con esta se debe ejecutar el proceso de parametrización, en donde se realiza la transferencia de datos del sistema de facturación de servicios públicos al aplicativo Android.

Para la opción Parametrización del menú principal, se diseñó la siguiente ventana Fig. 9.

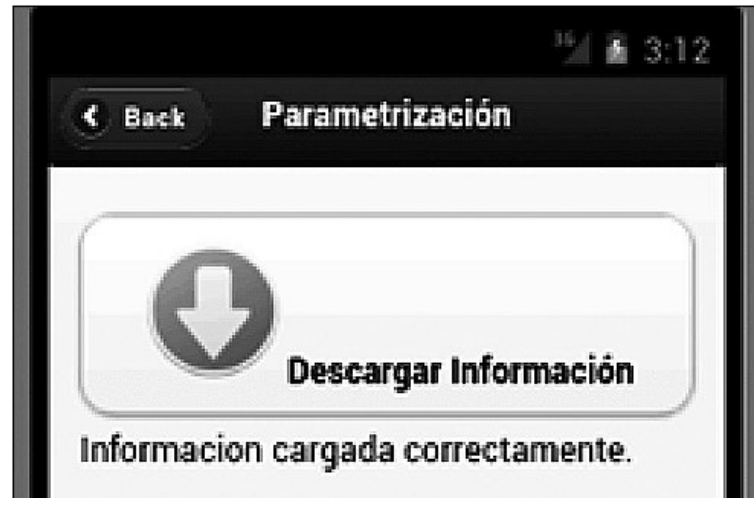

Fig. 9 Opción "Parametrización"

En dicha opción, a través del botón "Descargar Información", se procede a ejecutar la conexión vía internet con la base de datos central de la empresa de servicios públicos, de donde se obtiene la información de la ruta que debe seguir el aforador correspondiente, incluyendo: anomalías, estratos, usos y suscriptores. La información se transfiere en formato JSON, la cual se estructura como se muestra en la Fig. 10.

Una vez se realiza la conexión y se obtiene la información, el sistema muestra el aviso "Información cargada correctamente" indicando que ya se cuenta con la información requerida para empezar a realizar la toma de lecturas y el cálculo de facturación. 


\begin{tabular}{|c|c|c|c|}
\hline $\begin{array}{l}\text { 日10 } \\
\\
\text { 国 } 1 \\
\text { 田 } 2 \\
\text { 国 } 3 \\
\text { 国 } 4 \\
\text { 国 } 5 \\
\text { 国 } 6 \\
\text { 田 } 7\end{array}$ & 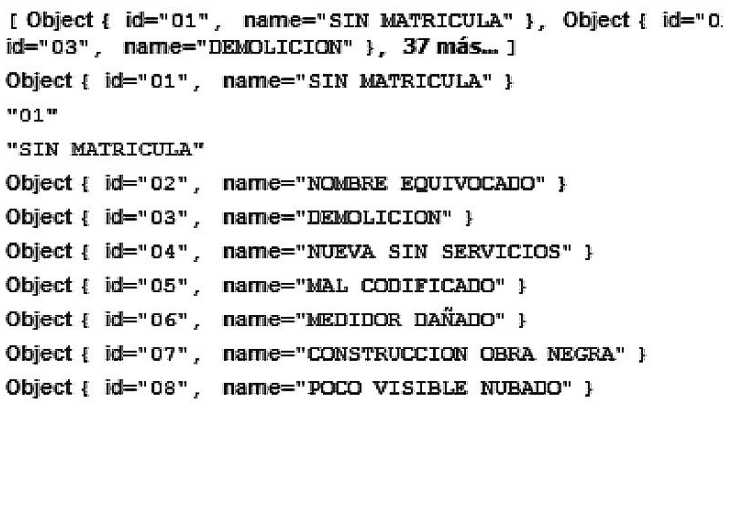 & $\begin{array}{ll}\text { @ } 0 & \\
& \text { direccion } \\
& \text { lectura } \\
& \text { medidor } \\
\text { nombre }\end{array}$ & 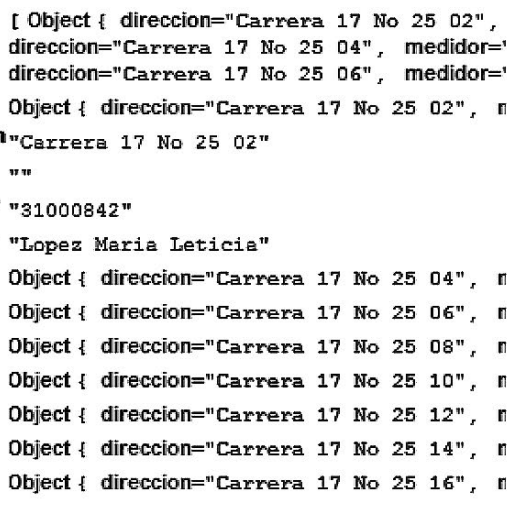 \\
\hline 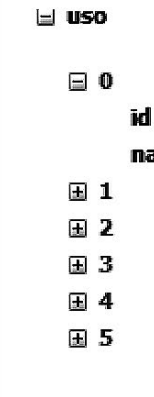 & 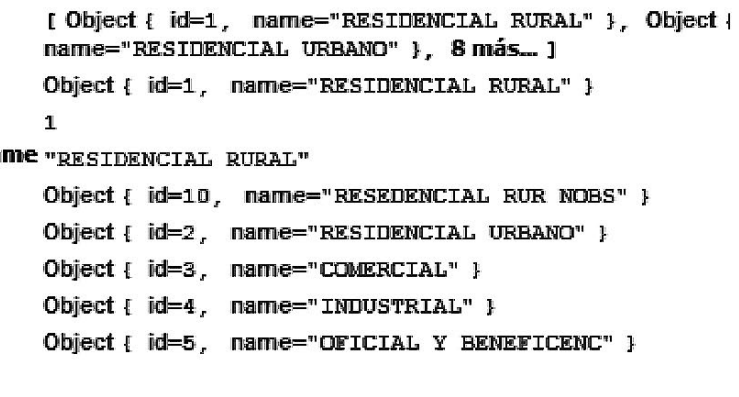 & $\begin{array}{l}\text { 曰0 } \\
\\
\end{array}$ & 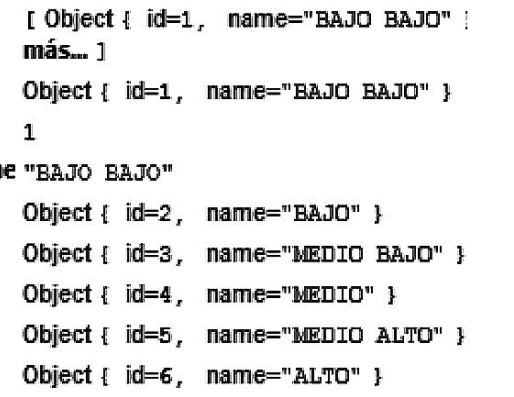 \\
\hline
\end{tabular}

Fig. 10 Información actualizada con la opción Parametrización

De esta forma, transferidos los registros, el aplicativo queda listo para la toma de lecturas.

Al ingresar a la opción Facturar, el sistema carga la pantalla que se muestra en la Fig. 11.

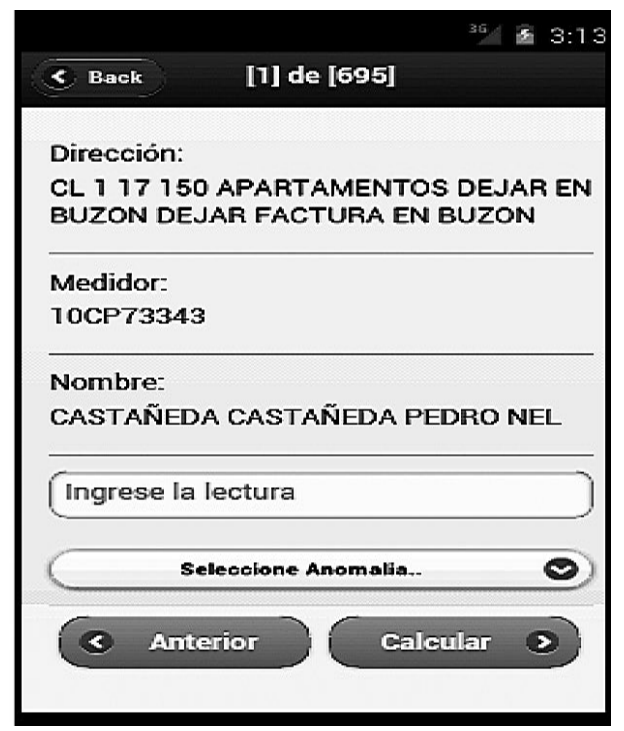

Fig. 11 Opción "Facturar"

En la figura anterior, se dan a conocer precisamente los datos que se deben registrar a la hora de la toma de la lectura del consumo en línea, que el aforador visualiza en el medidor. Es en esta opción se ejecuta el proceso central de la aplicación, el cual es "tomar lecturas del consumo del servicio, registrar novedades de aforo y actualizar y controlar en línea la información correspondiente de usuarios y facturación".

Si el aplicativo encuentra que ya se han tomado lecturas, se iniciará en la última lectura tomada.

Básicamente esta pantalla funciona de la siguiente forma:

- Se digita la lectura en la casilla designada "Ingrese la lectura", la cual debe corresponder al usuario y medidor que indica el sistema. Cuando inicia el aplicativo, el cursor se posiciona automáticamente en el cuadro de texto, "Ingrese la lectura".

- Si existe algún inconveniente en la toma de la lectura, se selecciona de la lista de anomalías, el problema que se ha presentado; si la lectura no se puede tomar, se digita la lectura con valor "0" y se registra. 
- Si no hay problemas de aforo, se oprime el botón Calcular. Si existe un problema de aforo y ya se seleccionó de la lista de anomalías, se aplica, de igual forma, la opción "Calcular."

- El botón "Calcular", además de ejecutar el proceso de cálculo de facturación del usuario al cual se le registró la lectura, permite que el sistema cargue de manera automática los datos del siguiente usuario de la ruta establecida.

- El botón "Anterior" permite que el sistema retroceda al usuario inmediatamente anterior,, en caso de precisar algún ajuste o cambio en los datos de aforo que le fueron tomados.

- No se puede registrar o modificar una lectura de un usuario anterior. Solo se pueden ajustar o cambiar los datos del aforo del usuario inmediatamente anterior al que se le está registrando las lecturas. Esto como sistema de control y seguridad en la captura e integridad en los datos.

En la Fig. 12 se observa la gráfica con la pantalla que muestra el resultado de un ejemplo del cálculo de la facturación de un suscriptor, una vez verificados los datos del usuario y registrados los datos de la lectura del respectivo aforo.

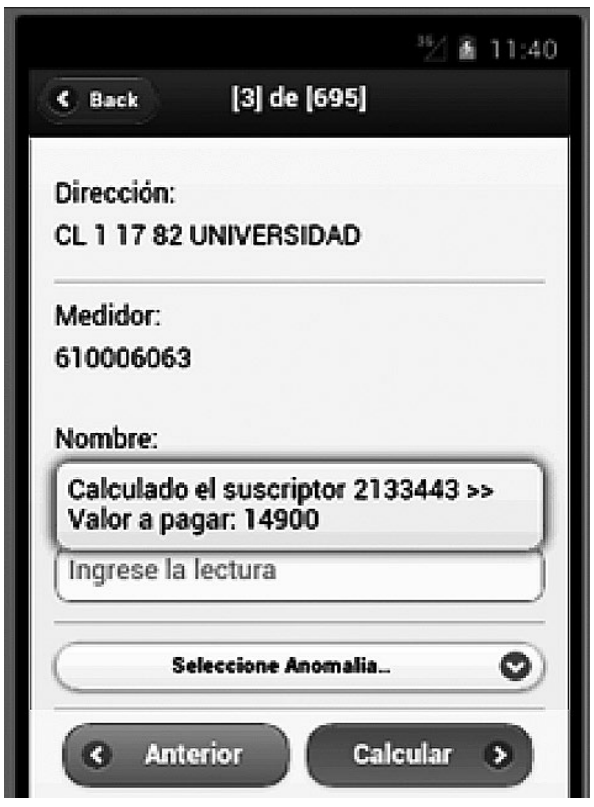

Fig. 12 Pantalla "Resultados del cálculo de facturación"
Es de anotar que a través del diseño del aplicativo tipo utilitario, se realizaron muchos ajustes, modificaciones y actualizaciones a las diversas pantallas, a las funcionalidades y a los procesos, de acuerdo con las pruebas del sistema que se iban realizando a medida que se diseñaba cada opción.

La tercera opción del menú es Ayuda, que a su vez contiene las opciones que se muestran en la Fig. 13.

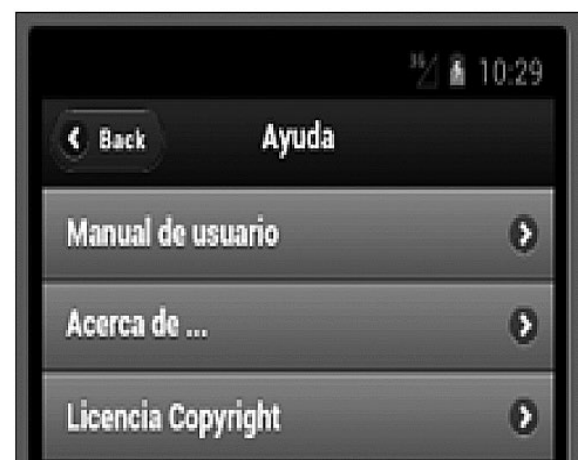

Fig. 13 Opción Ayuda

Manual del usuario, a través de la cual se accede a una página en donde se encuentra un instructivo general relacionado con el manejo de la aplicación y las diferentes opciones disponibles. La apariencia se muestra en la Fig. 14.

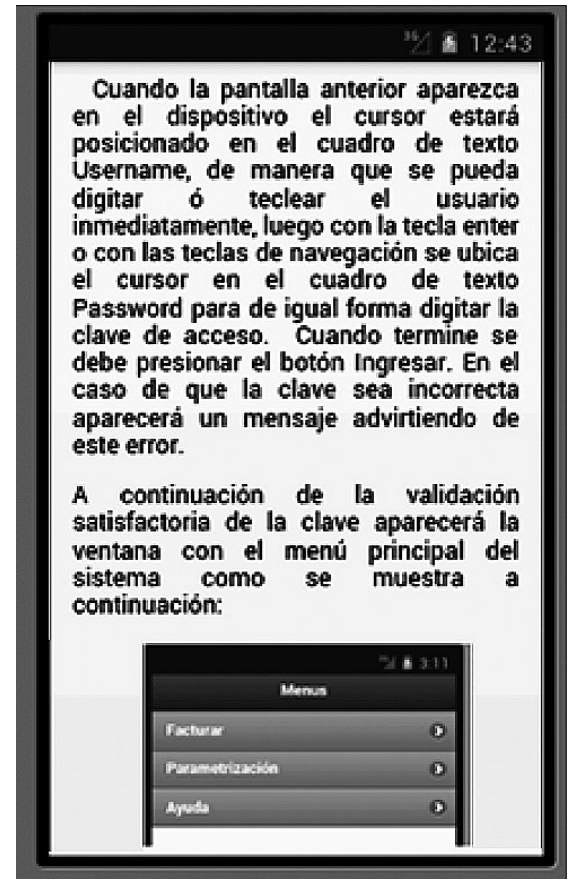

Fig. 14 Opción "Manual del usuario" 
Acerca de: contiene el nombre de la aplicación y la versión que se encuentra instalada en el dispositivo, al igual que la información de los desarrolladores con el fin de obtener asistencia en el funcionamiento de la aplicación, como se observa en la Fig. 15.

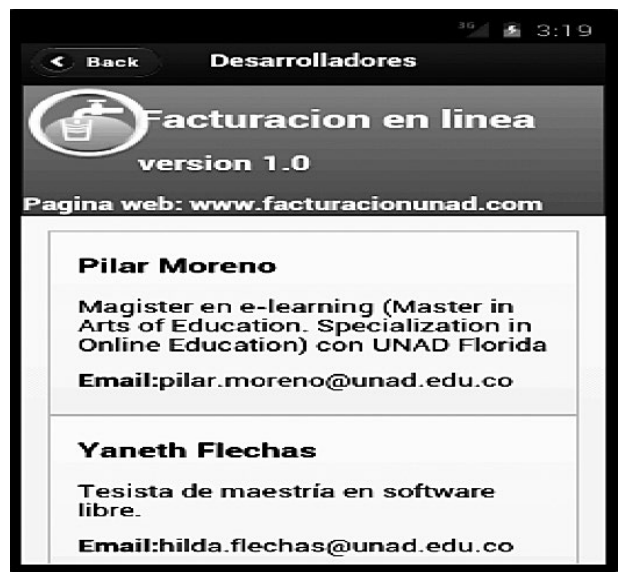

Fig. 15 Opción "Acerca de..."

Licencia Copyright: muestra los derechos de la aplicación y cuál es la licencia sobre la que se estableció el software. En la Fig. 16 se visualiza la información que despliega esta opción:

\section{10.30}

< Back

Licencia Copyri.

Q 2012. Reservados todos los derechos. Todos los titulos y derechos de autor correspondientes al "Sistema de lectura y cálculo de facturación en sitio a traves de dispositivos móviles" son propiedad de los autores, tienen derechos: reservados y està prohibido su uso. copia o distribución.

Autores:

Flechas Becertra Hilda Yanęth

Moreno Pilar Alexandra

Rojas López Carlos Alberto

Fig. 16 Opción "Licencia Copyright"

\section{Implementación del sistema}

El utilitario "Lectura en sitio", trabaja con una base de datos local de Android, la cual está montada en SQLite y contiene una única tabla donde se guardan los datos de autenticación; dicha tabla se llama USER y tiene la estructura,descrita en la Fig. 17.

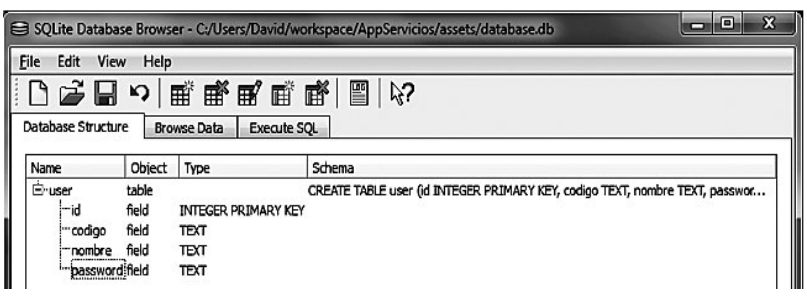

Fig. 17 Diseño tabla local del sistema en SQLite

SQLite es un sistema de gestión de bases de datos relacional compatible con ACID, contenida en una relativamente pequeña ( 275 kiB)2 biblioteca escrita en C. Así mismo, es un proyecto de dominio público creado por el doctor Richard Hipp. La biblioteca SQLite se enlaza con el programa pasando a ser parte integral del mismo. El programa utiliza la funcionalidad de SQLite a través de llamadas simples a subrutinas y funciones [3].

\section{El proceso de datos trabaja así:}

El usuario se identifica y la aplicación revisa en la base de datos local la información de autenticación; si son correctos ingresa a la aplicación. Luego, el usuario debe ejecutar el proceso de cargue de información; el utilitario de Android se conecta a un Servlet, que consiste en un aplique o pequeña aplicación Java (applet) que se ejecuta en un servidor web y se envía al usuario junto a una página web con objeto de realizar determinadas funciones, tales como el acceso a bases de datos o la personalización de dicha páginas web [4]. Este le proporciona la información de la ruta que el aforador debe seguir, con los datos de los suscriptores, a través de un archivo plano de rutas.

En el archivo de la ruta se encuentra el orden en que se debe tomar la lectura; es decir, el sistema va indicando, uno a uno, cada usuario al cual 
se le va a tomar lectura. Entonces, cuando termina de tomar una lectura. Continúa con el siguiente usuario de la lista, según la ruta.

El usuario comienza así el proceso de facturación: proporciona la lectura en el módulo y el sistema Android envía la lectura a otro Servlet, el cual realiza el cálculo de la facturación teniendo en cuenta la configuración y parametrización de la base de datos de la entidad de servicios públicos y, por último, devuelve el valor total de la facturación calculada.

La aplicación está disponible sólo en idioma Español. Para instalar la aplicación "Lectura en Sitio" para Android, se debe asegurar que la versión de Android sea compatible, así:

- Para teléfonos móviles, Android 2.1 o superior.

- Para tablets, Android 3.0 o superior.

- Para comprobar con qué versión cuenta el dispositivo, se debe acceder a Ajustes y seleccionar Acerca del teléfono o Acerca de la tablet. EI número de versión aparece en la sección "Versión de Android".

En cuanto a la conexión, se puede utilizar una red inalámbrica o la conexión 2G/3G/4G.

De esta forma, el utilitario es la aplicación encargada ,de establecer la conexión "en línea" o "en sitio". Con la base de datos de la empresa de servicios públicos, toma la lectura, la envía al sistema central en donde se hace el cálculo de la facturación y la devuelve nuevamente al aplicativo para ser visualizada, con la posibilidad posterior de imprimir la factura.

\section{Conclusiones}

Implementar el sistema aquí propuesto, como utilitario conectado al sistema de facturación de una empresa de servicios públicos, permite a dicha empresa optimizar la toma de lecturas en sitio y así obtener un mayor control del proceso y mejorar el servicio a sus usuarios.

Desarrollar el sistema presentado, como una aplicación móvil en Android, fue la mejor opción para cumplir con los requerimientos identificados y analizados para el proyecto software.

Utilizar el Modelamiento aquí propuesto permitió desarrollar una herramienta que se corresponda con los objetivos del proyecto y con los objetivos que debe tener un desarrollo de aplicaciones móviles en Android.

La sistematización del proceso realizado permite identificar los aspectos más relevantes que se deben tener en cuenta en cualquier desarrollo de una aplicación móvil, así como compartir los resultados y metodologías, como parte de la etapa de documentación que establece la ingeniería del software.

La principal ventaja del sistema desarrollado radica en que actúa como un módulo independiente que, si es requerido, estará enlazado con cualquier sistema de facturación de servicios públicos.

En cuanto a Android, como herramienta de programación usada, se resaltan grandes ventajas como: es software libre, existe toda una comunidad de desarrolladores para apoyo y soporte, es fácil de usar, tiene el apoyo de grandes empresas de hardware y móviles, ofrece precios competitivos, tiene acceso a aplicaciones- Apps y es compatible con gran variedad de dispositivos, incluyendo tablets. 
El almacenamiento de datos desde Android, se hizo mucho más sencillo utilizando la herramienta de base de datos SQlite, ya que es una base de datos relacional ligera, Open Source, que no demanda demasiados recursos y brinda un rendimiento adecuado para la tarea que se requiere frente al control de los usuarios de la aplicación y la toma de lecturas que se registran.

\section{Proyecciones y Aplicación}

El utilitario queda como sistema funcional para que sea conectado a cualquier sistema de facturación de servicios públicos, realizando primero un análisis de la data del sistema externo para, de esta forma, estandarizar el archivo de datos que se enviará al sistema de lectura y posteriormente se devolverá al sistema de facturación.

Se hace necesario, desarrollar un trabajo posterior que implemente más opciones que complementarían de una mejor forma esta aplicación, tales como:

- Permitir imprimir la factura en sitio.

- Realizar la contabilización de los conceptos facturados a la fecha.

- Permitir cargar un archivo con nuevos registros en los dispositivos móviles para la toma de lecturas, de la base de datos de servicios públicos almacenada en el servidor de la compañía.

- Generar informes que muestren en resumen los archivos planos generados para lectores digitales.
Nota: Las imágenes e ilustraciones utilizadas en este artículo, que no son inherentes al sistema, son propiedad de Android y Open Handset Alliance, de acuerdo con la licencia creative commons.

\section{Referencias}

[1] Sysman. Soluciones efectivas: http://www.sysman. com.co/Site

[2] Infotrack. Más desafíos, más movilidad: http://www. infotrack.com.co/site/

[3] Definición tomada de: http://es.wikipedia.org/wiki/ SQLite

[4] Definición tomada de: http://www.definicion.org/servlet

[5] Catalán, Curso Android- Desarrollo de aplicaciones móviles, Maestros del web, 2011.

[6] Gómez y S. Gómez Oliveros, Curso de programación en Android, Open Libra, 2011.

[7] M. Gargenta, Learning Android, O'Reilly Media, Inc., 2011.

[8] sgoliver.net blog. "Bases de Datos en Android (I): Primeros pasos". Recuperado de: http://www.sgoliver. net/blog/?p=1611

[9] SQLite. "Ejemplo de base de datos SQLite Android". Recuperado de: http://www.slideshare.net/mejiaff/ ejemplo-base-de-datos-sqlite-android

[10] Varios, Tecnología y Desarrollo en Dispositivos Móviles, UOC, 2011

[11] W.-M. Lee, Beginning Android Tablet Application Development, Wrox: Hoboken, NJ, USA , 2011.

[12] Z. Mednieks, Programming Android, 2nd Edition, O’Reilly Media, Inc, 2012. 